\title{
Efectos del aumento de peso y el riesgo de cáncer de mama
}

Dual effects of weight and weight gain on breast cancer risk Huang, Z.; Hankinson, S.; Colditz, G. et.al. Jama 1997; 278: 1407-1411

\section{Objetivo}

Evaluar el índice de masa corporal (IMC) a los 18 años y en la adultez así como el cambio en el peso corporal en relación al riesgo de cáncer de mama pre y postmenopaúsico (incidencia y mortalidad).

\section{Diseño}

Estudio de cohorte.

\section{Lugar}

Cuestionario enviado a enfermeras residentes en 11 estados de EE.UU.

\section{Pacientes}

Un total de 121700 enfermeras entre 30 y 55 años de edad respondieron un cuestionario, el cual fue actualizado cada 2 años, con el fin de identificar los nuevos casos de cáncer de mama y las variaciones en el peso. El seguimiento fue de 16 años.

Se excluyeron las mujeres con otros cánceres y con información incompleta sobre los factores de riesgo en el primer cuestionario. La cohorte analizada incluyó 95256 mujeres.

\section{Evaluación de los factores de riesgo}

Se obtuvo información sobre edad, estado menstrual (pre y postmenopaúsicas), peso actual, altura y otras ocho variables asociadas al riesgo de cáncer de mama, dentro de las que se incluyó el uso de reemplazo hormonal.

\section{Medición de resultados principales}

Con el peso y la altura se obtuvo el IMC el cual fue categorizado en 10 grupos para el cálculo de la incidencia, y en 5 para la mortalidad. Las variaciones en el peso se categorizaron en 5 grupos. Se estimó el riesgo relativo de cáncer de mama ajustado por las demás variables. Se calculó el porcentaje de riesgo atribuible* a la ganancia de peso, utilizadoras ocasionales o permanentes de hormonas vs no utilizadoras y una combinación de ambos (ganancia de $>2 \mathrm{~kg}$. y/o consumidora de hormonas vs ninguno de estos factores)

\section{Resultados Principales}

Se detectaron 2517 cánceres de mama (60\% postmenopáusicas) Entre las premenopáusicas, el riesgo relativo(RR) de cáncer de mama fue cercano a 1 cuando el IMC actual no superaba los $26 \mathrm{~kg} / \mathrm{m}^{2}$; pero se halló una correlación inversa en los grupos de IMC superior a $26 \mathrm{~kg} / \mathrm{m}^{2}$ (IMC $>31 \mathrm{~kg} / \mathrm{m}^{2}$ vs $\leq 20 \mathrm{~kg} / \mathrm{m}^{2}$, RR=0.58 [IC 95\% 0.42-0.80], $\mathrm{p}<0.001$ ).

En las postmenopáusicas no se observó una asociación significativa entre IMC e incidencia de cáncer de mama. Sin embargo en el subgrupo de mujeres que nunca usaron hormonas en la postmenopausia se halló una fuerte asociación entre ambas variables (IMC $>31$ $\mathrm{kg} / \mathrm{m}^{2}$ vs $\leq 20 \mathrm{~kg} / \mathrm{m}^{2}, \mathrm{RR}=1.59$ [IC 95\% 1.09-2.32], $\mathrm{p}<0.001$ ). No se observó un aumento de riesgo en las utilizadoras de hormonas. El IMC a los 18 años mostró una relación inversa con la incidencia de cáncer de mama en la pre y en la postmenopausia (IMC $>25 \mathrm{~kg} / \mathrm{m}^{2}$ vs $<18 \mathrm{~kg} / \mathrm{m}^{2}, R R=0.61$ [IC 95\% 0.45-0.83], $\mathrm{p}<0.001$ ).

El aumento de peso mostró el mismo comportamiento que el IMC en relación al estado menstrual y al consumo de hormonas. El RR de esta variable en las mujeres que nunca usaron hormonas fue de 1.99 (IC 95\% $1.43-2.76$ p 0.001) para el aumento de peso de más de $20 \mathrm{~kg}$. vs no modificación en el mismo.

El IMC y el aumento de peso exhibieron una fuerte correlación con la mortalidad por cáncer de mama de similar magnitud y asociación en las no utilizadoras de hormonas que el observado para la incidencia.

\section{Conclusiones}

Evitar el aumento de peso en la adultez puede contribuir significativamente a la prevención del cáncer de mama en esta etapa, especialmente entre las no utilizadoras de hormonas.

\section{COMENTARIO}

La mayoría de los trabajos publicados sobre el tema mostraron una relación inversa entre IMC a los 18 años e incidencia de cáncer de mama, y en general el sobrepeso en la postmenopausia mostró una débil asociación con la incidencia(1).

A diferencia de los otros, el presente estudio incluyó las variaciones en el peso cada 2 años, el momento del aumento de peso y el uso de reemplazo hormonal en la postmenopausia. De este modo permitió observar que el riesgo conferido por el aumento de peso en la menopausia se manifiesta principalmente en las mujeres no utilizadoras de hormonas.

El efecto contrario del peso en la premenopausia se atribuye a que la mujer obesa sufre de anovulación con mayor frecuencia y por ende se reducen los niveles de estradiol y progesterona sérica. En la postmenopausia el tejido adiposo convierte androstenediona en estrona y la disminución en la SHBG (Sex Hormone Binding Globulin) en mujeres obesas aumenta el estradiol disponible.

Dado el frecuente uso actual de tratamientos hormonales en la menopausia, el sobrepeso o el aumento de peso pierden valor en el riesgo de cáncer de mama. De acuerdo a los resultados de este importante estudio sería recomendable evitar el aumento de peso en mujeres no utilizadoras de hormonas. Por otra parte debería aconsejarse también a las pacientes con cáncer de mama evitar el sobrepeso, dado al aumento en la mortalidad exhibida.

Todos sabemos que abundan los motivos para desaconsejar y tratar el sobrepeso.

Este estudio muestra, dentro de la compleja interacción de factores que determinan riesgo para cáncer de mama, que el sobrepeso puede jugar algún rol en su incidencia.

*Ver Glosario

Dr. Claudio Lorusso

Servicio de Ginecología. Hospital Italiano de Buenos Aires. 